\title{
Research on Producing Pure Hydrogen by Chemical Chain Reaction Using Pyrolysis Gas from Agricultural Waste Based on $\mathrm{NiO} / \mathrm{NiAl}_{2} \mathrm{O}_{4}$ Oxygen Carrier
}

\author{
Yang Luhan, Chen Duotian, Zhong Jianjun \\ College of Materials and Energy, South China Agricultural University, Guangzhou, China
}

Email address:

291322019@qq.com (Yang Luhan),1165828006@qq.com (Chen Duotian),2467930190@qq.com (Zhong Jianjun)

\section{To cite this article:}

Yang Luhan, Chen Duotian, Zhong Jianjun. Research on Producing Pure Hydrogen by Chemical Chain Reaction Using Pyrolysis Gas from Agricultural Waste Based on $\mathrm{NiO} / \mathrm{NiAl}_{2} \mathrm{O}_{4}$ Oxygen Carrier. Science Discovery. Vol. 6, No. 3, 2018, pp. 164-170. doi: $10.11648 /$ j.sd.20180603.14

Received: April 6, 2018; Accepted: May 18, 2018; Published: June 23, 2018

\begin{abstract}
China produces a large number of agricultural wastes annually, most of which are treated by incineration, and the utilization rate is very low. This paper had constructed a hydrogen production system for chemical chain of agricultural and forestry waste, focusing on the effects of loading capacity, promoter and baking temperature on the oxidation and catalytic performance of $\mathrm{NiO} / \mathrm{NiAl}_{2} \mathrm{O}_{4}$ oxygen carrier and the characteristics of its reduction and physical properties. The results showed this oxygen carrier had good circulation ability. After many redox reactions, the output of $\mathrm{H}_{2}$ had no obvious attenuation, and $\mathrm{NiO}$ could be attached to the surface of $\mathrm{NiAl}_{2} \mathrm{O}_{4}$ effectively. Therefore, $\mathrm{NiO} / \mathrm{NiAl}_{2} \mathrm{O}_{4}$ oxygen carrier had certain mechanical properties, not only the pore was fine, but also the gas flew through the chain cycle reaction completely. The research of this paper has important significance for high-grade utilization of agricultural and forestry waste, environmental protection, energy saving and emission reduction.
\end{abstract}

Keywords: Agricultural and Forestry Waste, Chemical Chain Reaction, Oxygen Carrier, Hydrogen Production

\section{基于 $\mathrm{NiO} / \mathrm{NiAl}_{2} \mathrm{O}_{4}$ 氧载体的农废物热解气链式循环制纯氢研究}

\section{杨璐菡, 陈铎天, 钟健军}

材料与能源学院能源与环境系统工程系, 华南农业大学, 广州, 中国

邮箱:

291322019@qq.com（杨路菡）, 1165828006@qq.com(陈铎天),2467930190@qq.com(钟健军)

摘要: 中国年产大量农废物, 但多以焚烧处理, 利用率极低。本项目以利用农废物制高品位清洁能源 $\mathrm{H}_{2}$ 为目标, 构建 了农林废弃物热解气化学链制氢体系, 重点研究了负载量、助剂、烘焙温度对 $\mathrm{NiO} / \mathrm{NiAl}_{2} \mathrm{O}_{4}$ 氧载体氧化和催化性能的影 响, 以及其还原性能和物理表征的特点, 发现该氧载体具有较好的循环能力, 在经过多次氧化还原反应后, $\mathrm{H}_{2}$ 产量无 明显衰减, 并且 $\mathrm{NiO}$ 能够有效附着在 $\mathrm{NiAl}_{2} \mathrm{O}_{4}$ 表面上, 因此该氧载体具有一定机械性能, 不仅孔隙细淢, 更有利于气体 流经促进链式循环反应完全。该项目的研究对农废物高品位利用、环境保护、节能减排等方面具有重要意义。

关键词: 农林废弃物, 化学链式反应, 氧载体, 制氢 


\section{1. 引言}

传统化石能源在利用过程中, 产生大量的 $\mathrm{CO}_{2}$, 导致 温室效应明显，据2016年11月发布的《全球碳预算报告》 统计 [1], 2015年全球化石燃料及工业二氧化碳排放总量达 到363亿吨, 如何减少碳排放成为全球热门话题。

随着中国农业快速发展和天然林保护工程的实施，可 以能源化利用的生物质总量逐年递增, 目前每年可获得生 物质资源量达到 3.14 亿tce (吨煤当量), 其中秸秆为 1.70 亿tce, 薪材为 1.44 亿tce, 生物质能源有很大的开发潜力。 因此, 农林废弃物热解气化技术的研究和应用, 具有十分 重要的意义[2]。

2017年中国农业部发布 1 号文件[3]，提出推进农业供 给侧结构性改革实施意见。提出推进绿色发展, 增强农业 可持续发展能力, 全面推进农业废弃物资源化利用, 鼓励 各地加大农作物秸秆综合利用支持力度, 打好农业面源污 染防治攻坚战[4]。

天然气蒸汽重整制氢 (SMR) 由于技术成熟、产品相 对简单等优点, 仍然是目前工业上最主要的制氢方法 [5]。 该技术存在反应器内部温度较高, 需采用成本很高的耐高 温不锈钢材料, 同时水蒸气重整反应速度慢, 若要在制氢 过程中将 $\mathrm{CO}_{2}$ 分离出来需要大量的能耗的缺陷, 这对未来 大规模制氢并捕集 $\mathrm{CO}_{2}$ 是不利。而化学链制氢技术 ( CLH) 采用燃料与空气非混合技术, 具有 $\mathrm{CO}_{2}$ 内分离的特点, 并 且产品 $\mathrm{H}_{2}$ 纯度高且分离简单, 可以实现 $\mathrm{CO}_{2}$ 零排放, 受到 了人们越来越多的关注 [6-8]。

\section{2. 化学链制氢技术原理}

\section{1. $\mathrm{H}_{2}$ 的制取与 $\mathrm{CO}_{2}$ 的捕集}

化学链制氢装置由 3 个反应器组成, 整个过程按照 3 个步骤来进行 $\mathrm{H}_{2}$ 的制取及 $\mathrm{CO}_{2}$ 的捕集 [16]:

(1) 在还原反应器 (燃料反应器) 中, 燃料与氧载体发 生反应, 燃料被完全氧化为 $\mathrm{CO}_{2}$ 和 $\mathrm{H}_{2} \mathrm{O}$ (把水蒸气冷 凝下来即可得到纯净的 $\mathrm{CO}_{2}$ ), 同时氧载体被还原为 还原态:

(2) 还原态的氧载体进入制氢反应器 (蒸汽反应器) 中, 与通入的水蒸气发生反应产生 $\mathrm{H}_{2}$, 同时氧载体被部 分氧化;

(3) 部分氧化的氧载体进入氧化反应器 (空气反应器) 中, 利用热空气将其完全氧化。该空气氧化步骤除 了起到将氧载体完全氧化的作用外, 还可以除去反 应过程中产生的积碳等副产物。

(蒸汽反应器和空气反应器中氧载体均发生氧化反应, 所以在实际操作过程中, 常将空气反应器省略或将上述两 种反应器整合为同一反应器, 通过在其进气口加装三通阀 连接水蒸气和空气, 即可满足原本两种反应器的功能。)

\section{2. 氧载体的制备}

目前, 氧载体的制备与优化是化学链制氢的主要研究 方向之一。氧载体一般由供氧体和载体组成, 供氧体为过
渡金属的金属氧化物, 二者比例一般为 $20-80 \mathrm{wt} \%$ 。它能在 两个反应器之间循环使用, 反应既需要传递氧也需要传递 热量, 研发适用于不同燃料的高性能氧载体是化学链制氢 技术工业化的前提。基于化学链制氢过程的高效性以及经 济性, 氧载体要在燃料反应器和制氢反应器之间循环来完 成氧和能量的传递。为了使得燃料在燃料反应器中得以充 分转化, 氧载体的制备及其性能优化是化学链制氢系统大 规模应用的前提和基础, 这也是本项目研究的重点。

\subsection{1. 氧载体的选择}

目前比较受关注的活性组分是金属氧化物基氧载体, 一般来说, 氧载体的金属氧化物都是过渡元素, 主要是 $\mathrm{Ni}$ 、 $\mathrm{Fe} 、 \mathrm{Cu} 、 \mathrm{Co}$ 和 $\mathrm{Mn}$ 及其氧化物; 而惰性载体可以增强氧载 体的整体机械强度和抗烧结、磨损的能力, 维持颗粒内部 孔的结构。要使氧载体的反应性能达到最好, 活性组分与 惰性载体间存在一个最佳比例[9-11]。

\subsection{2. 制备工艺的选择}

目前氧载体的制备工艺有: 沉淀法、共沉淀法、机械 混合法、浸渍法、溶胶-凝胶法、分散法、喷雾干燥法等。 本项目主要选用共沉淀法、溶胶-凝胶法、浸渍法来制备 氧载体[12-14]。

\section{(1)溶胶凝胶法}

1. 将制备供氧体相关金属化合物和惰性载体相关原 料根据不同配比配制成溶液并摚拌均匀。

2. 使用磁力搅拌器设置一定温度边加热边搅拌上述 溶液, 直至大部分溶液蒸发, 形成凝胶后转入干燥箱以一 定温度干燥至干。

3. 将干燥后的氧载体放入马弗炉程序升温进行轱烧, 制备完成。

(2)浸渍球法

1. 将金属化合物与完整铝球（或研碎铝球）根据不同 质量比例混合, 加入一定量蒸馏水并将其密封放入超声波 清洗器处理均匀。

2. 固液经超声波处理完毕后转入鼓风干燥箱以一定 温度干燥。

3. 将干燥后的氧载体放入马弗炉程序升温进行㷽烧, 制备完成。

(3)共沉淀法

1. 将供氧体相关盐和惰性载体相关盐根据不同配比 配制成盐溶液并摚拌均匀。

2. 使用磁力搅拌器设置一定温度边加热边摚拌上述 溶液, 并以一定速率滴加氨水/碳酸铵至溶液中, 调节 $\mathrm{PH}$ 至合适值可析出沉淀物。

3. 静置冷却溶液至室温, 进行抽滤洗涤, 将滤饼转入 干燥箱以一定温度干燥至干。

4. 将干燥后的氧载体放入马弗炉程序升温进行煅烧, 制备完成。

方法分析:

1.溶胶凝胶法: 制法简便, 步骤简单, 且用料配比精 确, 但耗时较长; 制得的氧载体样品呈红褐色带晶体状, 成色美观, 硬度适中, 缺点是催化活性较弱。 
2.浸渍法：制法最简便，操作步骤最少，但是得到的 样品配比不够精确; 制得的氧载体样品呈红褐色球状/红 褐色大颗粒状, 硬度最大, 缺点是铝球/铝颗粒上附着的 活性物质粉末易脱落。

3.共沉淀法: 制法较简便, 虽 $\mathrm{pH}$ 调配难度大且具有不 确定性, 但是用料配比精确; 制得的氧载体样品呈砖红色 土粉状, 缺点是样品易碎成粉末。

\section{3. $\mathrm{NiAl}_{2} \mathrm{O}_{4}$ 负载 $\mathrm{NiO}$ 的氧载体氧化性能实验结果 与分析}

在本实验中, 各项参数设定为床层温度为 $850^{\circ} \mathrm{C}, \mathrm{N}_{2}$ 流量为 $90 \mathrm{ml} / \mathrm{min}, \mathrm{CH}_{4}$ 流量为 $10 \mathrm{ml} / \mathrm{min}$, 变量主要为负载 量、助剂、焙烧温度等, 主要比较 $\mathrm{CO}_{2}$ 平均百分比含量。

\section{1. 负载量对 $\mathrm{NiO} / \mathrm{NiAl}_{2} \mathrm{O}_{4}$ 氧载体氧化性能的影响}

在本实验中, 各项参数设定为床层温度为 $850^{\circ} \mathrm{C}$, $\mathrm{N} 2$ 流量为 $90 \mathrm{ml} / \mathrm{min}, \mathrm{CH}_{4}$ 流量为 $10 \mathrm{ml} / \mathrm{min}$, 焙烧温度 $900{ }^{\circ} \mathrm{C}$ 的条件下, 通过一系列实验研究负载量分别为 $20 \mathrm{wt} \% 、 40 \mathrm{wt} \% 、 60 \mathrm{wt} \% 、 80 \mathrm{wt} \%$ 制得的氧载体对 $\mathrm{CH}_{4}$ 化 学链燃烧的影响。

分析图1（a）、（b）、（c）、（d）可得，随着负 载量的提高, 催化剂的最大催化效率也随之提高, 在相同 $\mathrm{CH}_{4}$ 流速的情况下, 通气 $5 \mathrm{~min}$ 是负载量为 $20-80 \mathrm{wt} \%$ 的氧载 体对应的 $\mathrm{CO}_{2}$ 的气体相对百分比含量分别为 $74.95 \%$ 、 $91.21 \% 、 96.91 \%$ 和 $93.09 \%$, 可以看出, 除了负载量为 $80 \mathrm{wt} \%$ 时 $\mathrm{CO}_{2}$ 的相对百分比含量有所下降，其他几种都随着负载 量的提高而提高, 这表明随着负载量增加, 甲烷的转化率 先增加后降低, 在负载量为 $60 \mathrm{wt} \%$ 时达到最高, 转化率为 $96.90 \%$ 。

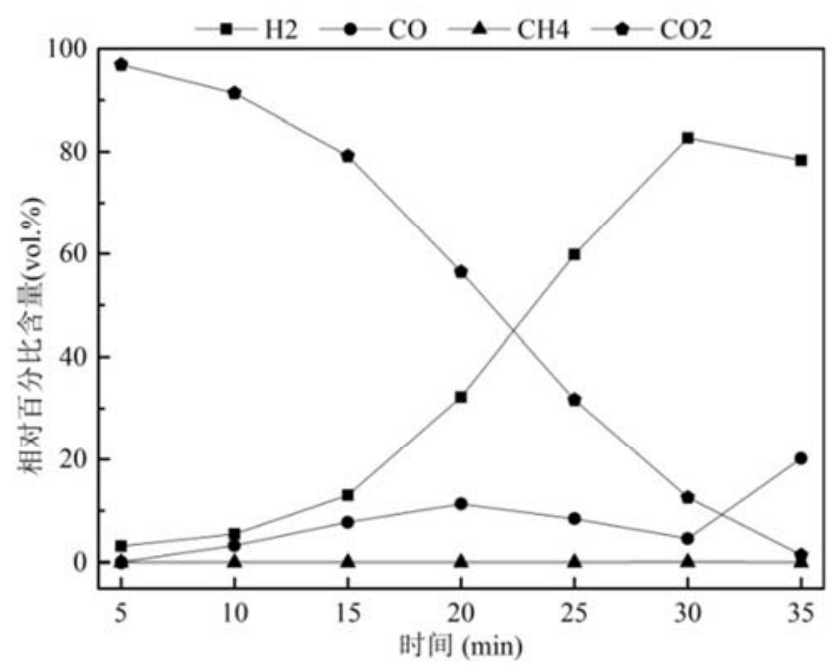

(a) 负载量 $80 \mathrm{wt} \%$

图1 负载量对气体组分的影响。
分析氧载体的持续时长可知, 负载量为 $20 \mathrm{wt} \%$ 的氧 载体反应到 $25 \mathrm{~min}$ 时, $\mathrm{CO}_{2}$ 的相对百分比含量已经降至 $1.44 \%$, 而对于负载量为 $40 \mathrm{wt} \%$ 氧载体, 降到同样水平的 $\mathrm{CO}_{2}$ 的相对百分比含量的时长为 $30 \mathrm{~min}$, 负载量为 $60 \mathrm{wt} \%$ 和 $80 \mathrm{wt} \%$ 的氧载体需要 $35 \mathrm{~min}$ 。

综合以上数据分析, 推测负载量不同导致氧载体催化 性能不同的原因, 可能是由于负载量过低时, 并不能充分 利用载体的孔隙, 导致催化效率低, 催化时长短, 但随着 负载量的增加, 也使得活性组分更加容易烧结和积碳, 因 此负载量 $60 \mathrm{wt} \%$ 和 $80 \mathrm{wt} \%$ 之间催化时长差距并不明显, 因 此综合经济效益进行考虑, 决定选择负载量为 $60 \mathrm{wt} \%$ 进行 下一步实验[15]。

\section{2. 助剂对 $\mathrm{NiO} / \mathrm{NiAl}_{2} \mathrm{O}_{4}$ 氧载体催化效果的影响}

在本实验中, 各项参数设定为床层温度为 $850^{\circ} \mathrm{C}, \mathrm{N}_{2}$ 流量为 $90 \mathrm{ml} / \mathrm{min}, \mathrm{CH}_{4}$ 流量为 $10 \mathrm{ml} / \mathrm{min}$, 负载量为 $60 \mathrm{wt} \%$, 焙烧温度 $900^{\circ} \mathrm{C}$ 的条件下, 改变助剂种类。

比较图2 (a)、(b)数据可知, 在甲烷转化率最高的时 候, 即反应时间为 $5 \mathrm{~min}$ 的时候, 助剂为 $\mathrm{Fe}$ 的氧载体 $\mathrm{CO}_{2}$ 的相对百分比含量 $98.71 \%$, 高于 $\mathrm{Mn}$ 的 $89.82 \%$; 随着反 应的进行, $\mathrm{NiO} / \mathrm{NiAl}_{2} \mathrm{O}_{4}$ 活性氧逐渐减少, 同时催化效 率也降低, $\mathrm{CH}_{4}$ 完全反应, 生成少量的 $\mathrm{H}_{2}, \mathrm{CO}$, 并随着 反应的进行 $\mathrm{H}_{2}$ 相对百分比含量越来越高; 在 $35 \mathrm{~min}$ 的时 候, 助剂为 $\mathrm{Mn}$ 的氧载体 $\mathrm{CO}_{2}$ 相对百分比含量为 $3.2 \%$, 略 高于 Fe的 $2.7 \%$ 。

综合以上数据并查阅文献可知, 铁基氧载体的反应 活性较低, 氧转移能力和甲烷转化能力都不强, 但具有 较强的抗积碳能力, 而镍基氧载体反应活性高, 但容易 积碳 [15], 因此在 $\mathrm{Fe}$ 的协同作用下, 提高 $\mathrm{NiO} / \mathrm{NiAl}_{2} \mathrm{O}_{4}$ 抗积碳能力, 延长催化剂寿命, $\mathrm{Mn}$ 虽然也有助于增强 氧载体抗烧结能力, 但由于 $\mathrm{Fe}$ 的成本较低, 而且对环境 无害, 因此综合各种因素考虑选用 $\mathrm{Fe}$ 为助剂进行后续实 验。

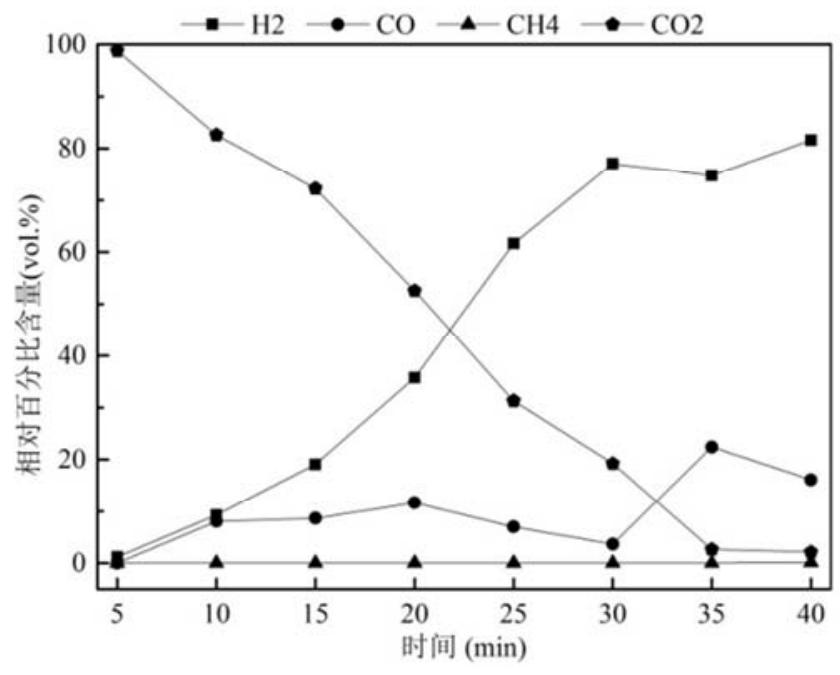

(a) 助剂 $\mathrm{Fe}$ 


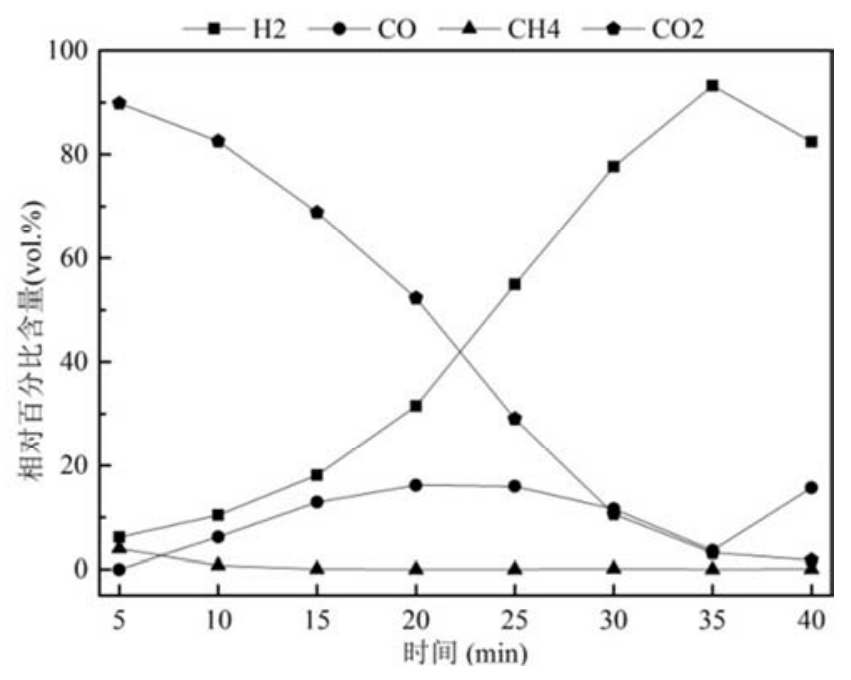

(b) 助剂 $\mathrm{Mn}$

图2 助剂对气体组分的影响。

\section{3. 焙烧温度对 $\mathrm{NiO} / \mathrm{NiAl}_{2} \mathrm{O}_{4}$ 氧载体催化效果的影响}

在本实验中, 各项参数设定为床层温度为 $850^{\circ} \mathrm{C}, \mathrm{N}_{2}$ 流量为 $90 \mathrm{ml} / \mathrm{min}, \mathrm{CH}_{4}$ 流量为 $10 \mathrm{ml} / \mathrm{min}$, 负载量为 $60 \mathrm{wt} \%$ 的条件下, 通过改变焙烧温度进行研究。

通过图3（a）、（b）、（c）、（d）可以看出，在 $900^{\circ} \mathrm{C}$ 焙烧得到的 $\mathrm{NiAl}_{2} \mathrm{O}_{4}$ 浸渍后制得的 $\mathrm{NiO} / \mathrm{NiAl}_{2} \mathrm{O}_{4}$ 具 有相对其他焙烧温度较高的催化活性, 最高 $\mathrm{CO}_{2}$ 的相对百 分比含量 $96.91 \%$, 而且反应到 $30 \mathrm{~min}$ 时 $\mathrm{CO}_{2}$ 的相对百分比 含量还有 $9.95 \%$, 相比之下, $600^{\circ} \mathrm{C} 、 700^{\circ} \mathrm{C}$ 和 $800^{\circ} \mathrm{C}$ 环境 下焙烧的，其持续时长比 $900^{\circ} \mathrm{C}$ 焙烧的短， $25 \mathrm{~min}$ 时 $\mathrm{CO}_{2}$ 的相对百分比含量分别为 $0.42 \% 、 4.45 \%$ 和 $11.63 \%$, 可以 看出, 随着焙烧温度的提高, $\mathrm{NiO} / \mathrm{NiAl}_{2} \mathrm{O}_{4}$ 催化效果越好。 $\mathrm{CH}_{4}$ 百分比含量几乎为 0 , 说明 $\mathrm{CH}_{4}$ 完全参与反应, 从 $\mathrm{CO}$ 的相对百分比含量比较明显可以推测, 随着反应的进行, 氧载体含氧量逐渐减少, $\mathrm{CH}_{4}$ 没能与 $\mathrm{NiO}$ 反应就发生了分 解反应导致碳沉积。

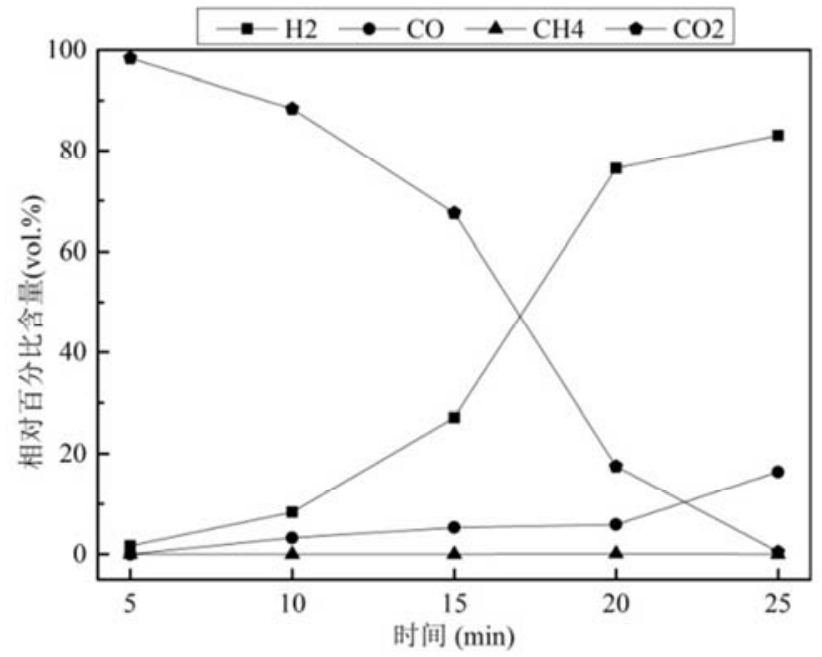

(a) 焙烧温度 $600^{\circ} \mathrm{C}$

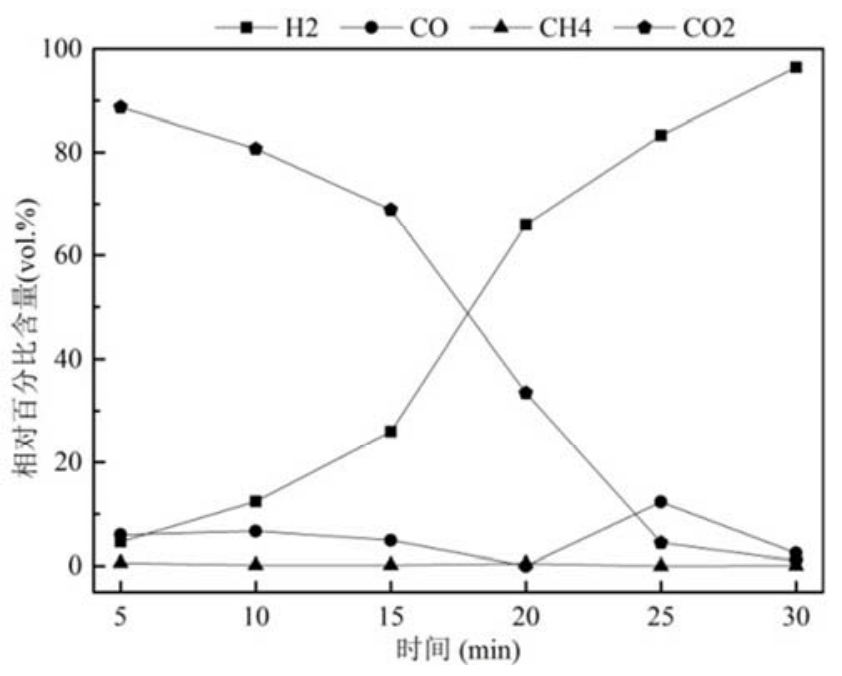

(b) 焙烧温度 $700^{\circ} \mathrm{C}$

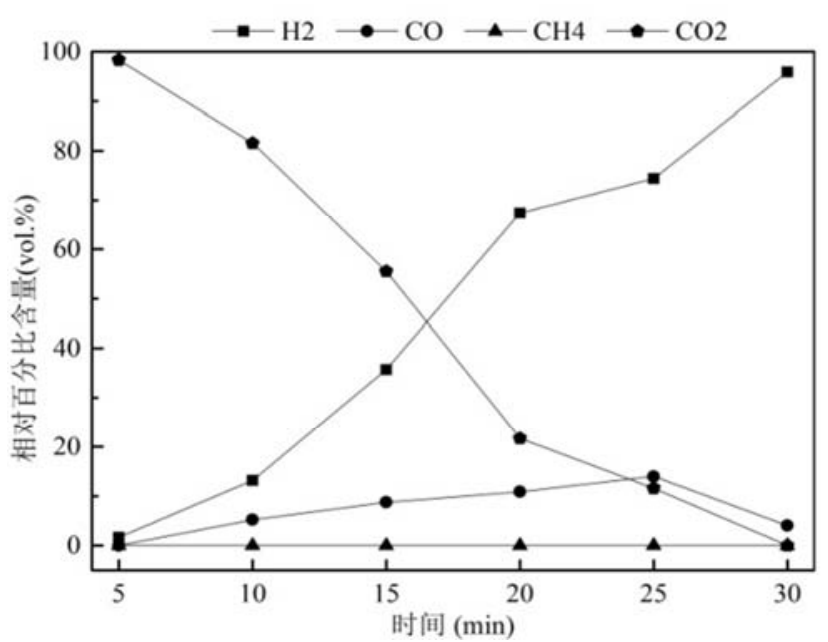

(c) 焙烧温度 $800^{\circ} \mathrm{C}$

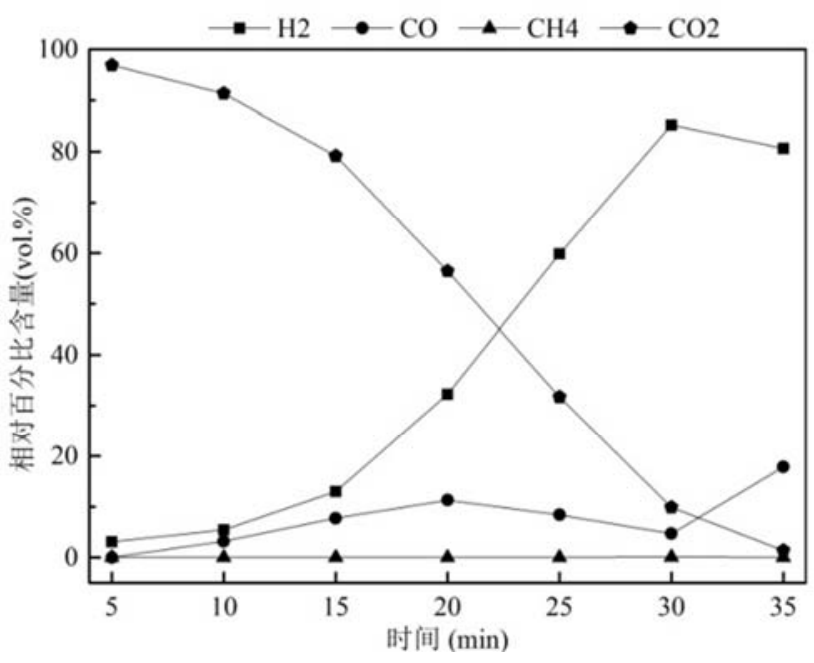

(d) 焙烧温度 $900^{\circ} \mathrm{C}$

图3 焙烧温度对气体组分的影响。

综合以上数据并查阅资料发现, 温度越高, $\mathrm{NiO}$ 和 $\mathrm{Al}_{2} \mathrm{O}_{3}$ 合成 $\mathrm{NiAl}_{2} \mathrm{O}_{4}$ 比例也越高, 因此推测 $600^{\circ} \mathrm{C}$ 焙烧的 
$\mathrm{NiAl}_{2} \mathrm{O}_{4}$, 在反应温度为 $850^{\circ} \mathrm{C}$ 的固定床中, $\mathrm{NiO}$ 和 $\mathrm{Al}_{2} \mathrm{O}_{3}$ 继续 $\mathrm{NiAl}_{2} \mathrm{O}_{4}$, 降低了催化剂活性组分的比例, 从而降低 整个氧载体的反应活性。而直接 $900^{\circ} \mathrm{C}$ 焙烧可以提前使 $\mathrm{NiO}$ 和 $\mathrm{Al}_{2} \mathrm{O}_{3}$ 向 $\mathrm{NiAl}_{2} \mathrm{O}_{4}$ 转化, 尽可能降低在试验过程中 $\mathrm{NiO}$ 向 $\mathrm{NiAl}_{2} \mathrm{O}_{4}$ 转化, 提高催化效率和性能。

\section{4. $\mathrm{NiO} / \mathrm{NiAl}_{2} \mathrm{O}_{4}$ 的还原态氧载体还原性能研究}

在本实验中, 各项参数设定为床层温度为 $750^{\circ} \mathrm{C}, \mathrm{N}_{2}$ 流量为 $90 \mathrm{ml} / \mathrm{min}, \mathrm{H}_{2} \mathrm{O}(\mathrm{g})$ 流量为 $10 \mathrm{ml} / \mathrm{min}$, 主要比较各 次反应生成 $\mathrm{H}_{2}$ 的平均百分比含量。

反应进行每隔 2 分钟收集 1 次气体，每次反应共收集 5 次气体; 对氧载体进行多次氧化还原反应, 并分别在各次
反应中收集气体, 以检测还原态氧载体在多次反应过后制 氢性能的衰退情况。

表1中展示了氧载体在经过第1次、5次、10次氧化还 原反应后, 通入 $\mathrm{H}_{2} \mathrm{O}(\mathrm{g})$ 制 $\mathrm{H}_{2}$ 过程中, $\mathrm{H}_{2}$ 的产量变化。 通过表 2 可知，在第 10 次反应经过 $4 \mathrm{~min}$ 时最高 $\mathrm{H}_{2}$ 的相对百 分比含量为 $96.09 \%, \mathrm{H}_{2}$ 纯度高; $8 \mathrm{~min}$ 内 $\mathrm{H}_{2}$ 含量保持在 $80 \%$ 左右; 而 $8 \mathrm{~min}$ 后 $\mathrm{H}_{2}$ 开始大幅下降, 可知此时还原态氧载 体已被大量氧化并逐渐失活。排除积炭等因素影响, 氧 载体在经过多次氧化还原反应后, $\mathrm{H}_{2}$ 产量并没有明显衰 减现象, 可知还原态氧载体的还原性能良好, 具有较好 的持续循环能力。

表1 氧化还原次数对 $\mathrm{H}_{2}$ 产量的影响。

\begin{tabular}{|c|c|c|c|c|c|}
\hline 反应时间 & $2 \min$ & $4 \mathrm{~min}$ & $6 \mathrm{~min}$ & $8 \min$ & $10 \mathrm{~min}$ \\
\hline 氧化还原循环次数 & $\mathrm{H}_{2}$ 的相 $\bar{X}$ & & & & \\
\hline 第1次 & $96.37 \%$ & $96.02 \%$ & $83.29 \%$ & $85.04 \%$ & $77.54 \%$ \\
\hline 第10次 & $96.02 \%$ & $85.01 \%$ & $80.11 \%$ & $78.54 \%$ & $71.67 \%$ \\
\hline 第20次 & $94.63 \%$ & $96.09 \%$ & $92.37 \%$ & $79.12 \%$ & $75.92 \%$ \\
\hline
\end{tabular}

\section{5. $\mathrm{NiAl}_{2} \mathrm{O}_{4}$ 负载 $\mathrm{NiO}$ 的氧载体物理表征}

本项目氧载体的物相组成测试由 $\mathrm{X}$ 射线衍射仪完成 [17]。主要目的是分析氧载体的晶体结构以及对其物相进 行定性和定量分析; 原理是在一定波长的 $\mathrm{X}$ 射线照射下,
每种晶体物质都只产生自己特有的衍射特征峰。图4为X 射线衍射仪处理分析系统分析得到的衍射图, 分析衍射图 上的衍射峰值可知, 样品的物相只由 $\mathrm{NiO}$ 和 $\mathrm{NiAl}_{2} \mathrm{O}_{4}$ 结构组 成而没有其他物质参杂。

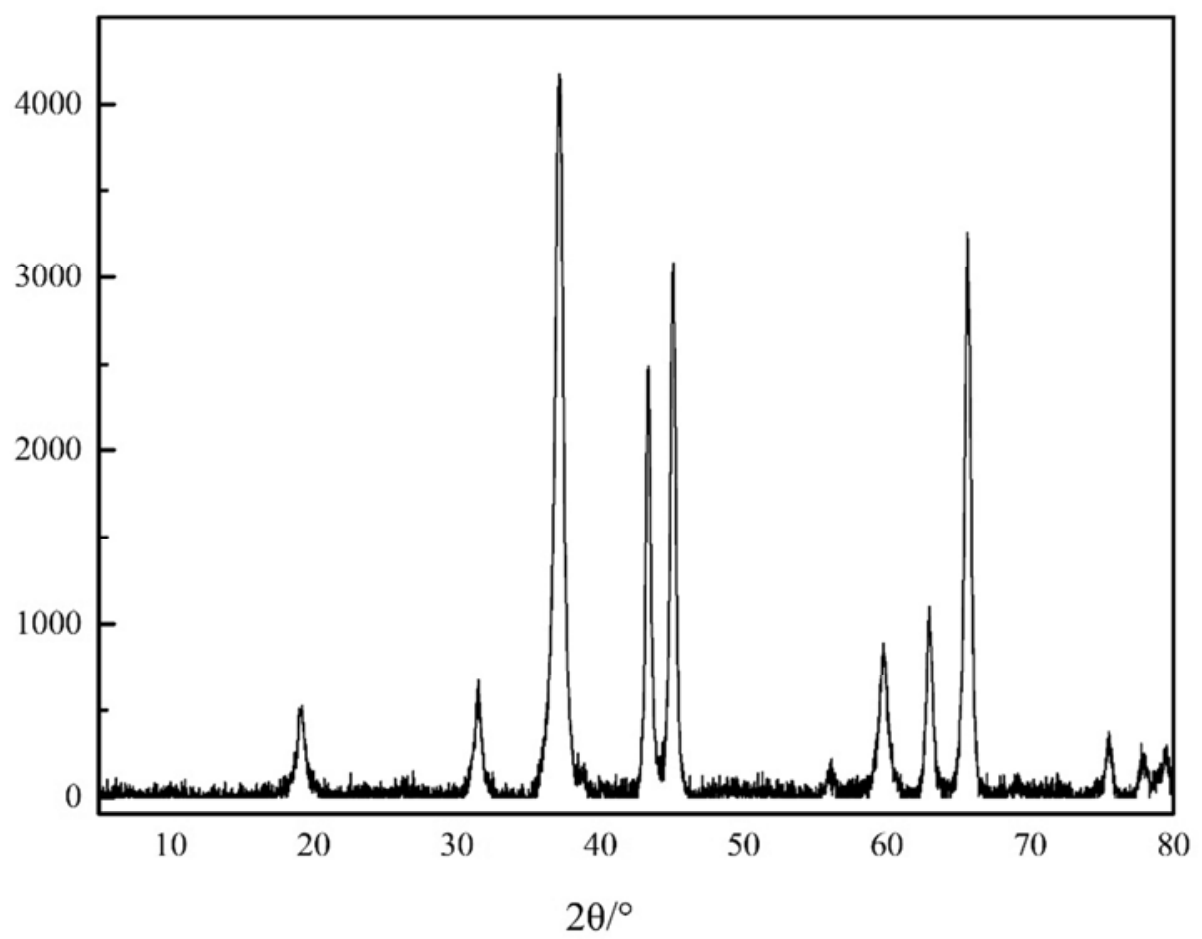

图4 X射线衍射分析图。

对氧载体的电镜扫描在场发射扫描电镜分析仪器上进行，主要观察氧载体表面的结构形貌和各部分的细节。图5

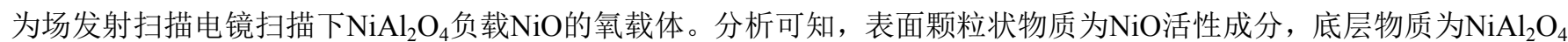
惰性载体。 $\mathrm{NiO}$ 能够有效附着在 $\mathrm{NiAl}_{2} \mathrm{O}_{4}$ 表面上, 其颗粒物大小适中, 分布均匀, 颗粒与颗粒间空隙较小; $\mathrm{NiAl}_{2} \mathrm{O}_{4}$ 结构 稳定, 具有一定机械性能, 减少反应损耗, 且孔隙细淢, 有利于气体流经并反应完全。 

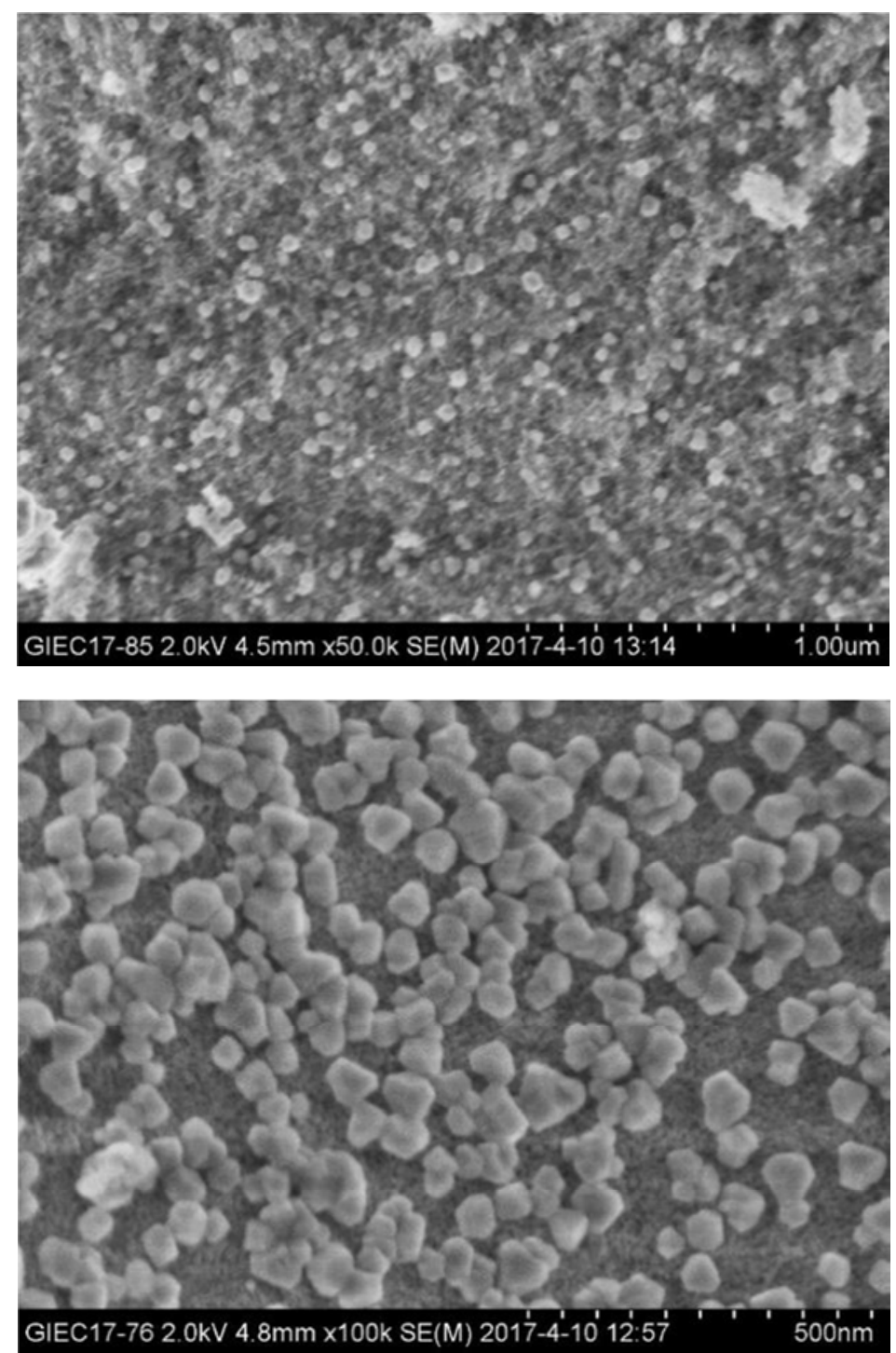

图5 电镜扫描下的 $\mathrm{NiO} / \mathrm{NiAl}_{2} \mathrm{O}_{4}$ 氧载体。

\section{4. 结论}

相较于以金属氧化物作为惰性载体的传统氧载体, 本 项目以尖晶石为载体的氧载体, 通过控制相应的合成条件 以获得具有硬度较高的介孔结构的镍铝尖晶石, 并负载氧 化镍作为主要供氧体, 而尖晶石同样可起到辅助供氧的作 用, 形成双供氧体系。故对比传统氧载体, 本氧载体具有 更好的机械稳定性和化学活性。

针对农林废弃物利用率低的现状，提出了利用农林废 弃物热解挥发份化学链循环重整制纯 $\mathrm{H}_{2}$ 协同 $\mathrm{CO}_{2}$ 减排的 技术路线, 实现农林废弃物的能源化利用; 利用氧载体的 反应特性, 免去了氢气复杂的分离提纯步骤, 也能更简单 实现二氧化碳的收集、提纯与捕获, 实现纯氢气的制备和 纯二氧化碳的捕捉, 达到节能减排的功效。本研究对农林 废弃物连续热解产物链式循环制纯氢气协同二氧化碳减 排关键技术的研究具有长远良好的发展前景, 对促进农林 经济发展和环境保护具有重大意义。

\section{致谢}

本文为国家自然科学基金（51706074），广东省科技 计划项目（2015B020237010、2016A020221028）的阶段 性成果之一。

\section{参考文献}

[1] 陈婉.《2018年度全球碳市场进展报告》发布全球碳市场呈 现新格局[J].环境经济,2018(05):44-47。

[2] 关海滨,张卫杰,范晓旭,赵保峰,孙荣峰,姜建国,董红海,薛旭 方. 生物质气化技术研究进展 [J]. 山东科 学,2017,30(04):58-66。

[3] 农业部关于推进农业供给侧结构性改革的实施意见[J].饲 料研究,2017(05):46-52。 
[4] 王红彦,王飞,孙仁华,高春雨,王亚静,孙宁,王否,毕于运.国外 农作物秸秆利用政策法规综述及其经验启示 $[\mathrm{J}]$.农业工程 学报,2016,32(16):216-222。

[5] 王斯晗,张㻦健.天然气蒸汽重整制氢技术研究现状 [J].工业 催化,2016,24(04):26-30。

[6] 孙兆松,梁皓,尹泽群.化学链制氢技术研究进展[J].化学工业 与工程,2015,32(05):71-78。

[7] 曾亮,巩金龙.化学链重整直接制氢技术进展 [J].化工学 报,2015,66(08): 2854-2862。

[8] 罗明,王树众,王龙飞,吕明明,肖仲正,朱佳斌.基于化学链技 术制氢的研究进展 [J].化工进展,2014,33(05):1123-1133。

[9] 史奇良,陈时熠,薛志鹏, 王东, 向文国.铁基载氧体化学链制 氢特性实验研究[J].中国电机工程学报,2011,31(S1):168-174。

[10] 胡月.生物质废物化学链制氢工艺中铁基载氧体制备与性 能研究[D].清华大学,2014。

[11] 祝捷.生物质废物热解气单塔固定床化学链制氢系统实验 研究[D].清华大学,2015。
[12] 蒋林林, 赵海波, 张少华, 等.煤基化学链燃烧技术的 $\mathrm{Fe} 2 \mathrm{O} 3 / \mathrm{A} 12 \mathrm{O} 3$ 氧载体研究 [J]. 工程热物理学报, 2010 , (06):1053-1056。

[13] 梅道锋, 赵海波, 马兆军, 等. $\mathrm{Fe}_{2} \mathrm{O}_{3} / \mathrm{Al}_{2} \mathrm{O}_{3}$ 氧载体制备方 法的研究 [J]. 燃料化学学报, 2012, (07):795-802。

[14] 刘自松, 魏永刚, 李孔斋, 等. $\mathrm{Fe}_{2} \mathrm{O}_{3} / \mathrm{Al}_{2} \mathrm{O}_{3}$ 氧载体用于甲 烷化学链燃烧: 负载量与制备方法的影响 $[\mathrm{J}]$. 燃料化学学报, 2013，(11):1384-1392。

[15] Ming Luo, Shuzhong Wang, Longfei Wang, et al. Reduction kinetics of iron-based oxygen carriers using methane for chemical-looping combustion[J]. Journal of Power Sources. 2014.

[16] H.P. Hamers, F. Gallucci, P.D. Cobden, et al. CLC in packed beds using syngas and $\mathrm{CuO} / \mathrm{Al}_{2} \mathrm{O}_{3}$ :Model description and experimental validation[J]. Applied Energy. 2014.

[17] Xing Cai, Xiaohong Wang, Xin Guo, et al. Mechanism study of reaction between $\mathrm{CO}$ and $\mathrm{NiO}$ surface during chemical-looping combustion: Role of oxygen[J]. Chemical Engineering Journal. 2014. 